\title{
Uso de medicamentos para glaucoma durante a gravidez $e$ amamentação.
}

\author{
Maria Luiza Viana Maia (1)
}

\section{CARTA AO EDITOR}

Embora tenhamos literatura sobre opções de tratamento para glaucoma em gestantes, as dúvidas sobre qual tratamento médico pode ser mais seguro e o manejo adequado do glaucoma durante a gravidez persistem até hoje ${ }^{1}$. Em uma pesquisa publicada em 2007 na revista Eye, 26\% dos entrevistados relataram já ter tratado uma mulher grávida com glaucoma. Esta situação teoricamente infrequente está se tornando menos frequente e na prática clínica diária encontramos mulheres em idade fértil com glaucoma, que levantam preocupações sobre o tratamento de que necessitam para sua doença e possíveis complicações durante a gravidez e a amamentação. Os avanços no tratamento médico e cirúrgico do glaucoma congênito e infantil têm contribuído para isso, pois têm permitido que os pacientes cheguem à idade adulta com boa função visual.

Estima-se que durante a gravidez a PIO seja reduzida em até $10 \%$, sendo essa diminuição mais pronunciada no terceiro trimestre. A causa parece ser multifatorial, sendo a alteração hormonal a mais importante e que condiciona o aumento do fluxo de saída do humor aquoso e a diminuição da pressão venosa episcleral ${ }^{2}$. No entanto, a evolução do glaucoma durante a gravidez é variável, apesar desse fator protetor hormonal teórico ${ }^{3,4}$. A maioria das pacientes permanece estável durante a gravidez, enquanto uma pequena porcentagem, aproximadamente $10 \%$, pode apresentar aumento da PIO ou progressão da doença ${ }^{3}$.

A impossibilidade de realizar estudos faz com que tenhamos que recorrer a séries de casos clínicos para obter mais informações sobre o manejo do glaucoma durante a gravidez. Em um estudo retrospectivo em 28 olhos de 15 mulheres publicado há 6 anos, 57,1\% dos olhos estudados (16 olhos no total) não progrediram e mantiveram a PIO estável durante a gravidez ${ }^{4}$ e apesar dessa tendência natural de diminuir a PIO, casos foram descrito em que 
houve progressão da doença durante a gravidez ${ }^{3-7}$. Muitas dessas mulheres são diagnosticadas com glaucoma congênito e glaucoma da infância ou que são desencadeados no desenvolvimento. Outros têm glaucoma inflamatório ou pigmentar. Em muitos casos, encontramos mulheres que têm uma redução significativa no campo visual em pelo menos um dos 2 olhos e que foram submetidas a várias intervenções cirúrgicas. É possível que nesses tipos de glaucoma, que são os que mais ocorrem na idade fértil, o comportamento da PIO não seja o mesmo observado no glaucoma primário de ângulo aberto ou em mulheres saudáveis nas quais a PIO foi estudada. Comportamento da PIO durante a gravidez.

Uma das dificuldades no tratamento do glaucoma na gravidez é a necessidade de manter a função visual em pacientes com defeitos de campo visual avançados, à luz da consideração cuidadosa dos riscos potenciais do tratamento médico ou cirúrgico, tanto para a mãe quanto para o paciente.

A decisão de tratar ou não e o tipo de medicamento a utilizar envolve individualizar cada caso. As opções de tratamento disponíveis no glaucoma (médico, trabeculoplastia a laser ou tratamento cirúrgico) são mais limitadas nesses casos. Seria aconselhável antecipar ao máximo a gravidez e explicar à paciente a importância de notificar o oftalmologista o quanto antes para controlar a PIO com o menor número possível de colírios.

Não existem estudos que garantam $100 \%$ de segurança do feto. Por esse motivo, recomenda-se a suspensão do tratamento médico no primeiro trimestre, período de maior risco para malformações fetais.

Nos casos em que seja necessário o estabelecimento de tratamento médico, tanto os efeitos colaterais que podem ocorrer pela passagem do fármaco para o feto ao cruzar a barreira hemato-placentária quanto os possíveis efeitos na motilidade uterina e o consequente risco de prematuridade nascimento ou aborto. De acordo com a classificação de segurança com base em modelos experimentais da FDA ( Tabela 1), a brimonidina pertence à categoria $B$, ou seja, nenhum efeito adverso no feto foi demonstrado em estudos em animais. Não existem estudos em humanos. $O$ restante dos medicamentos antiglaucoma (prostaglandinas, $\beta$-bloqueadores, inibidores da anidrase carbônica, colinérgicos ...) pertencem à categoria $\mathrm{C}$, ou seja, efeitos adversos no feto foram demonstrados em estudos controlados em animais, embora não existam estudos ou existam nenhuma evidência em humanos. A brimonidina pode ser considerada a droga mais segura durante a gravidez, pois é a única incluída na categoria B. No entanto, essa droga não apenas atravessa a barreira hematoencefálica, produzindo depressão do SNC e apneia em crianças pequenas, mas, além disso, pode atravessar a barreira hemato-placentária. 


\begin{tabular}{|c|c|}
\hline Categoría & Prior surgery \\
\hline A & $\begin{array}{l}\text { No se ha demostrado riesgo para el feto en } \\
\text { estudios controlados en humanos }\end{array}$ \\
\hline B & $\begin{array}{l}\text { No se ha demostrado riesgo para el feto en } \\
\text { estudios en animales gestantes o bien los que se } \\
\text { han demostrado no se han confirmado en } \\
\text { estudios en humanos }\end{array}$ \\
\hline C & $\begin{array}{l}\text { Se ha demostrado efecto teratogénico para el } \\
\text { feto en estudios en animales gestantes. Solo } \\
\text { debe usarse en caso de que los beneficios para la } \\
\text { madre superen los riesgos potenciales para el } \\
\text { feto }\end{array}$ \\
\hline D & $\begin{array}{l}\text { Existe clara evidencia de riesgo teratogénico. Los } \\
\text { beneficios podrian hacer aceptable su uso } \\
\text { durante el embarazo a pesar del riesgo en caso } \\
\text { de enfermedad grave y ausencia de alternativas } \\
\text { de tratamiento }\end{array}$ \\
\hline $\mathrm{x}$ & $\begin{array}{l}\text { Se han demostrado anomalias en el feto en seres } \\
\text { humanos o en animales. Los fármacos incluidos } \\
\text { en este grupo están contraindicados en el } \\
\text { embarazo }\end{array}$ \\
\hline
\end{tabular}

Os medicamentos de primeira linha no glaucoma, as prostaglandinas, pertencem ao grupo dos medicamentos da categoria C. Os análogos da prostaglandina F2 $\alpha$ têm atividade ocitócica e luteolítica e podem predispor ao aborto espontâneo ${ }^{8,9}$, embora estudos experimentais em animais não tenham encontrado efeito no embrião com doses até 15 vezes superiores à terapêutica em humanos ${ }^{10}$ Apesar de existirem séries de casos em que o uso de latanoprost durante a gravidez não foi associado a partos prematuros ou abortos ${ }^{11}$, a capacidade de atravessar a barreira hemato-placentária e o fato de poder afetar a motilidade uterina com os riscos que isso implica desaconselhar seu uso durante a gravidez.

Os $\beta$-bloqueadores tópicos podem causar bradicardia e arritmias no feto. No entanto, há anos os especialistas em obstetrícia usam $\beta$-bloqueadores como fármacos antihipertensivos na hipertensão desenvolvida durante a gravidez ${ }^{12,13}$. Sua forma de gel comercial com concentração menor $(0,1 \%)$ é uma opção de tratamento mais segura. Devido à maior experiência no uso desses medicamentos durante a gestação, consideramos ser o medicamento de primeira escolha.

O tratamento oral com inibidores da anidrase carbônica foi associado ao desenvolvimento de teratomas sacrococcígeos no recém-nascido, embora nenhum efeito adverso tenha sido relatado com o tratamento tópico. Recentemente, foi descrito retardo de crescimento intrauterino com necessidade de cesariana em uma mulher com glaucoma congênito que manteve o tratamento tópico durante a gravidez com a combinação fixa de timolol-dorzolamida ${ }^{3}$.

Em nosso centro, procuramos manter o paciente sem tratamento tópico durante o primeiro trimestre para evitar o risco de teratogênese. Nos casos em que o tratamento é necessário pelo risco de progressão, a primeira opção terapêutica é o $\beta$-bloqueador tópico, 
preferencialmente o timolol em sua formulação em gel, seguido dos inibidores tópicos da anidrase carbônica. Sempre que possível, mantemos a paciente sob observação e sem tratamento médico ou com o menor número de medicamentos possível durante o primeiro trimestre e no último mês de gravidez. Em todos os casos, descartamos o uso de prostaglandinas porque, apesar de haver estudos retrospectivos em que nenhum efeito colateral foi demonstrado para o feto.

Alguns autores acreditam que a pouca informação sobre a segurança dos medicamentos anti-hipertensivos durante a abordagem terapêutica da gravidez torna outra necessidade de incluir tratamento a laser ou cirurgia.

A trabeculoplastia a laser permite que a PIO seja mantida dentro dos limites normais com menos drogas hipotensoras. Pode ser uma boa alternativa de tratamento desde que a morfologia do ângulo o permita, algo incomum nos tipos de glaucoma que as mulheres em idade fértil apresentam. A trabeculoplastia a laser não é tão eficaz nesses casos devido às alterações angulares presentes, inerentes à própria doença, ou pela presença de sinéquias angulares. Os glaucomas inflamatórios congênitos ou desenvolvidos durante a infância como resultado de malformações na câmara anterior, como síndrome de Rieger, síndrome de Peters, síndrome de Axenfel ou aniridia tendem a ter ângulo comprometido, portanto os resultados de ALT ou SLT são mais limitados.

O uso de ciclodestruição de diodo na gravidez foi recentemente descrito. O objetivo do tratamento seria reduzir a PIO com o menor número de drogas possível antes de planejar a gravidez ${ }^{6}$. O tratamento pode ser feito com anestesia local e pode ser repetido em caso de controle insuficiente da PIO. As diferenças anatômicas em termos de morfologia e posição do corpo ciliar nos glaucomas congênitos e desenvolvidos na infância devem ser levadas em consideração, bem como as possíveis complicações em pacientes com escleras delgadas ou glaucomas inflamatórios.

A dificuldade cirúrgica nestes casos é maior, pois frequentemente nos encontramos em pacientes que foram submetidos a operações repetidas e com um compromisso angular que limita o tipo de cirurgia. No caso de picos hipertensivos, o risco de perda visual requer a decisão de filtrar o tratamento cirúrgico com anestesia local e evitar antimetabólitos. Por outro lado, é aconselhável manter o paciente em decúbito lateral para evitar compressão da veia cava e refluxo gastroesofágico, principalmente no terceiro trimestre.

Em relação ao uso de hipotensores durante a lactação, sabemos que sua passagem para o leite materno foi demonstrada ${ }^{14,15}$. Em relação aos hipotensores mais seguros durante a lactação, consideramos as mesmas opções terapêuticas que aplicamos durante a gravidez, utilizando timolol gel como medicamento de primeira linha. Para reduzir a quantidade de medicamento que passa para o recém-nascido, o colírio pode ser instilado imediatamente após a ingestão e o ponto lacrimal ocluído por 5 minutos, embora seja aconselhável suspender 
a amamentação se houver necessidade de tratamento antiglaucoma.

Em resumo, nos casos em que o tratamento médico é necessário, os prós e os contras do tratamento devem ser avaliados adequadamente em uma base individual, sem tratamento hipotensivo durante o primeiro trimestre, e apenas os medicamentos mais seguros para a mãe e o bebê devem ser considerados feto, $\beta$-bloqueadores tópicos e inibidores da anidrase carbônica realizando a oclusão do ponto lacrimal para reduzir a absorção sistêmica. É aconselhável interromper o tratamento médico semanas antes da data prevista para o parto.

Instituição afiliada: 1- Hospital metropolitano de Belém

Dados da publicação: Artigo recebido em 01 de Julho, revisado em 19 de Julho, aceito para publicação em 25 de Julho e publicado em 30 de Julho.

DOI: https://doi.org/10.36557/2674-8169.2021v3n7p01-02

Éber Coelho Paraguassu dr.paraguassu@periodicosbrasil.com.br

This work is licensed under a Creative Commons Attribution 4.0 International

License.

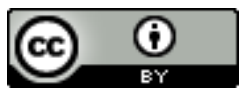

\section{REFERÊNCIAS}

1. Vaideanu D, Fraser S. Gestão do glaucoma na gravidez: um inquérito por questionário. Olho. 2007; 21: 341-2. [ Links ]

2. Ziai N, Ory SJ, Khan AR, Brubaker RF. Beta-gonadotrofina coriônica humana, progesterona e dinâmica aquosa durante a gravidez. Arch Ophthalmol. 1994; 112: 8016. [ Links ]

3. Mendez-Hernandez C, Garcia-Feijoo J, Saenz-Frances F, Santos Bueso E, Martinez-de-laCasa JM, Valverde Megias A, et al. Efeitos da terapia tópica de pressão intraocular na gravidez. Oftalmologia Clínica. 2012; 6: 1629-32. [ Links ]

4. Brauner SC, Chen TC, Hutchinson BT, Chang MA, Pasquale LR, Grosskreutz CL. O curso do glaucoma durante a gravidez: uma série de casos retrospectivos. Arch Ophthalmol. 2006; 124: 1089-94. [ Links ]

5. Johnson SM, Martinez M, Freedman S. Management of glaucoma in pregnation and lactation. Surv Ophthalmol. 2001; 45: 449-54. [ Links ]

6. Wertheim M, Broadway DC. Terapia a laser ciclodiodo para controlar a pressão 
intraocular durante a gravidez. Br J Ophthalmol. 2002; 86: 1318-9. [ Links ]

7. Coleman AL, Mosaed S, Kamal D. Medical therapy in pregning. J Glaucoma. 2005; 14: 414-6. [ Links ]

8. Salamalekis E, Kassanos D, Hassiakos D, Chrelias C, Ghristodoulakos G. Administração intra / extra-amniótica de prostaglandina F2a em morte fetal, abortos perdidos e terapêuticos. Clin Exp Obstet Gynecol. 1990; 17: 17-21. [ Links ]

9. Lipitz S, Grisaru D, Libshiz A., Rotstein Z, Schiff E, Lidor A, et al. Prostaglandina F2 alfa intraamniótica para interrupção da gravidez no segundo e no início do terceiro trimestre da gravidez. J Reprod Med. 1997; 42: 235-8. [ L Links ]

10. Chang $M C$, Hunt DM. Efeito da prostaglandina F2 alfa na gravidez precoce de coelhos. Natureza. 1972; 236: 120-1. [ Links ]

11. De Santis M, Lucchese A, Carducci B, Cavalieri A, de Santis L, exposição Merota A. Latanoprost na gravidez. Am J Ophthalmol. 2004; 138: 305-6. [ Links ]

12. Wide-Swensson D, Montal S, Ingemarsson I. Como obstetras suecos gerenciam distúrbios hipertensivos na gravidez: um estudo por questionário. Acta Obstet Gynecol Scand. 1994; 73: 619-24. [ L Links ]

13. Gladstone GR, Hordof A, Gersony WM. Administração de propranolol durante a gravidez: efeitos no feto. J Pediatr. 1975; 86: 962-4. [ L Links ]

14. Fidler J, Smith V, de Swiet M. Exccretion of oxprenolol and timolol in breast milk. Ir. J Obstet Gynaecol. 1983; 90: 961-5. [ Links ]

15. Salminen L. Revisão: absorção sistêmica de drogas oculares aplicadas topicamente em humanos. J Ocul Pharmacol. 1990; 6: 243-9. [ Links ] 